What's Up, DOC?

\section{Antipsychotic drugs and}

\section{mortality among elderly}

\section{patients}

Atypical antipsychotic drugs have been linked to increased mortality among elderly patients. Researchers examined data from almost 23000 patients over 65 years of age to see whether a similar risk existed for conventional antipsychotic drugs such as phenothiazines and butyrophenones. They found that, compared with atypical drugs, there was a $37 \%$ higher risk of death after up to I80 days of taking conventional agents (relative risk [RR] I.37, 95\% confidence interval [CI] I.27-I.49), regardless of patient age, sex, comorbidities and use of health care services that were likely predictors of death. The risk increased with the dose and was greater in the first 40 days of use. These results, if confirmed, suggest that conventional antipsychotic drugs are at least as likely as atypical agents to increase the risk of death among elderly patients and should not be used to replace atypical agents. Lower doses appear to be preferable. $N$ Engl J Med 2005;353:2335-4I

\section{The end of $\beta$-blockers?}

A meta-analysis examining the use of $\beta$-blockers as first-line therapy for hypertension found that the relative risk of stroke was $\mathrm{I} 6 \%$ higher for $\beta$-blockers (RR I.I6, 95\% CI I.04-I.30) than for other antihypertensive drugs. The risk of myocardial infarction (MI) and death due to any cause was the same for all drugs. When $\beta$-blockers were compared with placebo or no treatment, their use reduced the risk of stroke by I9\% (RR I.I9, 95\% CI I.07I.29), but again there was no difference in MI or all-cause death. Although $\beta$ blockers are used successfully for secondary prevention of coronary artery disease, these findings suggest that they should not be used alone for primary prevention and treatment of hypertension. Some patients taking $\beta$ need them for other reasons, but other patients can change to alternative, lowcost antihypertensive agents, although this should be done slowly to prevent rebound angina and other adverse events. Lancet 2005;366:1545-53

\section{Counselling versus drugs}

\section{for weight loss}

In this study, 224 obese adults, of whom $80 \%$ were women, received sibutramine (an appetite suppressant), lifestyle-modification group counselling ( 30 sessions), sibutramine and group counselling, or sibutramine with brief counselling given by a primary care physician over 8 visits. All groups were given the same diet and exercise regime. After one year, people receiving sibutramine and group counselling lost the most weight (mean I2.I kg, SD [standard deviation] 9.8 $\mathrm{kg}$ ), followed by sibutramine with brief counselling $(7.5 \mathrm{~kg}, \mathrm{SD} 8.0 \mathrm{~kg})$, group counselling alone $(6.7 \mathrm{~kg}, \mathrm{SD} 7.9 \mathrm{~kg})$ and sibutramine alone $(5 \mathrm{~kg}, \mathrm{SD}$ $7.4 \mathrm{~kg}$ ). Adverse events did not differ among the groups. These results suggest that sibutramine and intensive counselling together can lead to almost double the weight loss of those using sibutramine or counselling alone. They also confirm that lifestyle modification alone leads to significantly greater weight loss than use of blockers for hypertension will also

sibutramine alone. The findings provide strong support that weight-loss medications be used only in conjunction with comprehensive diet, exercise and behavioural advice. $N$ Engl J Med 2005;353:2III-20

\section{Too much iron?}

Iron tablets have well-known sideeffects. It is thus of interest to know whether low doses are as effective as high doses in treating iron-deficiency anemia in elderly patients ( $>80$ years). Ninety patients with iron-deficiency anemia received $\mathrm{I} 5,50$ or $150 \mathrm{mg}$ of elemental iron and were followed for 60 days. Patients with vitamin BI2 deficiency, severe illness or renal failure, malignant disease, active gastrointestinal blood loss or acute infection were excluded from the study, as were those who had received a blood transfusion in the week before enrollment. During the 2-month follow-up, the increase in hemoglobin and ferritin levels was significant and similar in all 3 groups; however, adverse effects - usually reported as abdominal pain, nausea or vomiting - were dose-dependent. Low-dose iron ( $15 \mathrm{mg} / \mathrm{d}$ ) is an effective option for elderly patients with irondeficiency anemia and does not have the side effects of higher doses. Am J Med 2005;II8:II42-7 — Compiled by Sally Murray, $C M A J$

DOI:I0.1503/cmaj.060059

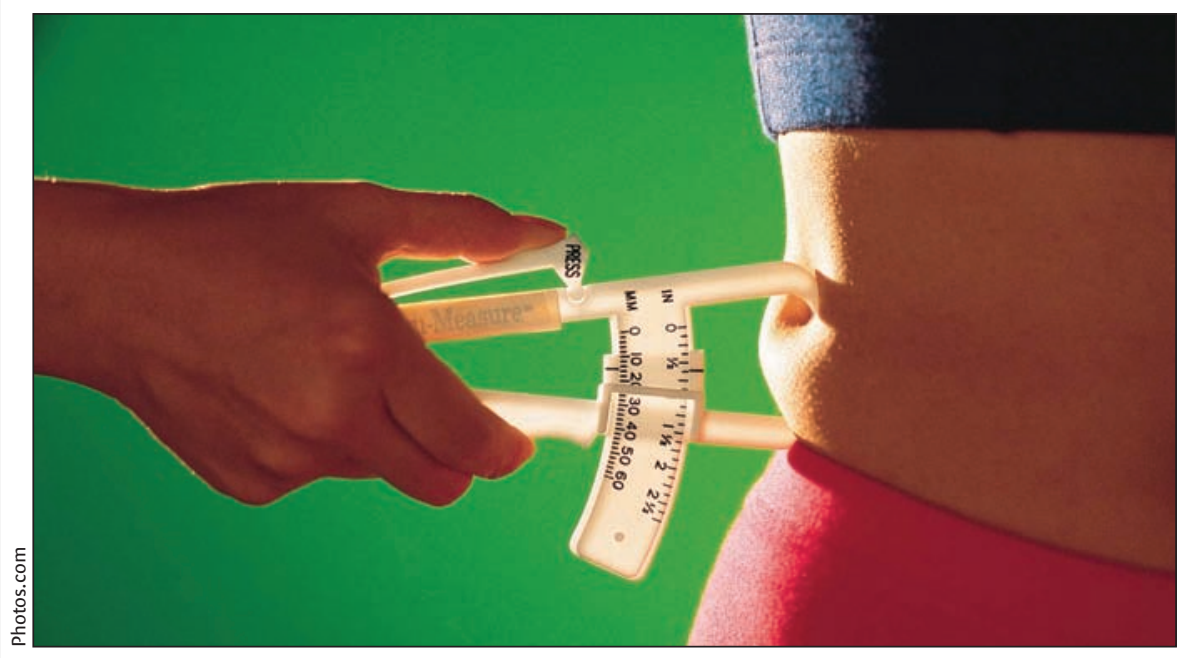

Talking it off 